\title{
INTRODUCTION BY THE EDITORS
}

This special issue of AJIS focuses on the importance of information systems and technology to small- and medium-sized enterprises (SMEs). The papers appearing in this issue were originally presented at the Second International Conference on SMEs in a Global Economy, which was held at the University of Wollongong in July 2002. All papers were double-blind refereed. The theme of the conference, which was organised by the Centre for SME Research and Development in the Faculty of Commerce, University of Wollongong, was "Sustaining SME Innovation, Competitiveness and Development in the Global Economy".

The conference built on continuing and increasing interest, from business, government and academic quarters, on the role of SMEs in their economies. In the APEC countries, SMEs typically comprise more than 90 percent of the business enterprises, and account for more than half of employment, and more than three-quarters of employment growth. They produce half of total output, and are responsible for about a third of exports. SMEs therefore comprise a vital part of their economies, and are the source of much innovation and entrepreneurial energy.

While the papers presented at the conference reflect the wide range of academic research into SMEs, the five papers included in this issue indicate something of a concentration on the actual and potential benefits for SMEs of information and communications technology (see the paper by Beal and Moha Asri) and electronic commerce (see the papers by Poon; Vrazalic et al.; and Jones et al.). Traditionally at a competitive disadvantage because of their lack of size and access to finance and markets, many SMEs, particularly those in the knowledge economy, are now finding that the competitive environment has tilted towards a more level playing field where they can compete on a more equal footing. In the emerging industries of the knowledge economy, entry costs are dramatically reduced and size matters less. The emerging research indicates that the factors that matter are a firm's knowledge base (see the paper by Al-Hawari and Hasan) and its ability to harness and build on it to innovate effectively. Although the benefits of utilizing ICT seem undeniable, research also shows that generally SMEs are having difficulty implementing ICT solutions and translating these into successful business strategies. While the authors clearly flag the need for more research in the area, their work here represent useful inputs into the continuing inquiry.

Charles Harvie and Boon-Chye Lee

Editors 